Classification

Physics Abstracts

$06.50-36.20-42.30-61.16 \mathrm{D}$

\title{
Three-dimensional reconstruction at the molecular level
}

\author{
Joachim Frank
}

Wadsworth Center for Laboratories and Research, New York State Department of Health, Albany, New York 12201-0509, U.S.A.

School of Public Health, State University of New York at Albany, 1400 Washington Ave., Albany, New York 12222, U.S.A.

(Received October 03, 1991; accepted April 09, 1992)

\begin{abstract}
This article gives a brief overview over the problem of obtaining three-dimensional images of macromolecules that occur in the form of single particles. The method of random-conical data collection and reconstruction, which has been successfully used for a variety of complex macromolecular assemblies such as the ribosome and the calcium release channel, receives particular attention. The roles of classification and restoration is obtaining artifact-free reconstructions, as well as the feasibility of merging different reconstructions are discussed both for negatively stained and ice-embedded macromolecules.
\end{abstract}

\section{Introduction.}

The electron microscope plays an increasingly important role in three-dimensional (3D) imaging of biological structures in all size ranges. It is perhaps not widely known that the method of computerized 3D reconstruction was pioneered in the field of electron microscopy (DeRosier and Klug, 1968), long before it was implemented as a routine diagnostic tool in medicine: most notably in Computerized Axial Tomography ("CAT-scan") and Magnetic Resonance Imaging (MRI).

The two size ranges to be distinguished (see Frank, 1989) pertain to (i) structures that are subcellular components, such as organelles of the cytoskeleton and (ii) in a much higher resolution range, macromolecules and macromolecular assemblies, such as ribosomes. This distinction is of practical importance because in the former category we have structures that are not necessarily reproducible in the strict sense of spatial congruence, even though they follow the same basic building plan. In the latter category, by contrast, most structures are very well defined and given the same chemical environment - may exist as identical "copies" of the same structure in a defined configuration. Thus only the latter type of specimens allow very effective methods of data collection at reduced doses of irradiation, designed to avoid serious problems of radiation damage. Accordingly, the types of experiment used in the two size ranges to obtain 3D information are quite different. Another important difference lies in the increased thickness of the specimens in which the subcellular structures are prepared (normally by plastic enbedment), which requires electron voltages in the intermediate $(200-400 \mathrm{kV})$ or high range (above $400 \mathrm{kV}$ ). 
This article will put its focus on the 3D imaging of macromolecules, in particular of those that come in the form of "single particles" and lack symmetries. The structure of biological macromolecules is intrinsically related to their function. Currently, a large data bank of protein structures is accumulating at a rapid rate, thanks to the progress in $x$-ray crystallography of protein crystals. However, many biological structures beyond a certain size range, mostly products of a complex assemblage of macromolecules ("macromolecular assemblies"), pose difficulties to the xray crystallography approach that preclude routine application of this technique. With increasing size, these structures are harder to crystallize, and - closely related to this difficulty - the crystals obtained in successful attempts may not be as well ordered as single-component structures. In fact, it is probably safe to say that generally, with increasing number of heterogeneous components, the number of conformational states of the entire assembly increases, and it becomes more and more difficult to describe this composite structure by any method that involves global averaging. This leaves a large "niche" for 3D imaging with the electron microscope.

Examples for such large multi-component structures are ribosomes and the calcium release channel of the sarcoplasmic reticulum.

\section{Principle of three-dimensional reconstruction.}

The electron microscope produces a parallel projection of the specimen in the plane of the film. When the negative staining technique is used, the stain completely dominates the image, and the molecule appears as a negative image - as an "exclusion of stain" - allowing its shape but little interior detail to be recognized. When the newer technique of frozen-hydrated electron microscopy (see Dubochet et al., 1988) is used, the image can be directly interpreted in terms of the projection of the potential of the molecule in its hydrated form, rendering all the its interior features faithfully $\left({ }^{1}\right)$.

For the 3D structure of the molecule to be retrieved, a number of electron microscopic projections must be available that cover a sufficiently large angular range. From these projections, the molecule may be reconstructed by one of the following techniques: weighted back-projection, Fourier reconstruction, or iterative real-space back-projection (see Zwick and Zeitler, 1973). Of these, the weighted back-projection method (Gilbert, 1972) has found most widespread use because of its computational efficiency. Essentially the same algorithm is used in many applications of medical tomography.

Because of the high susceptibility of biological macromolecules to radiation damage - even in negatively stained preparations -, the means of data collection is of crucial importance. Owing to the steady accumulation of electron dose in the course of the experiment, multiple projections obtained from a single macromolecule form an intrinsically inconsistent data set: for instance, two projections at different angles depict a particle in different states of structural degradation. Earlier attempts to reconstruct macromolecules in three dimensions based on such experiments (e.g. Hoppe et al., 1974) were handicapped by this problem.

Radiation damage can be reduced when the macromolecule either consists of many identical components (e.g. the building blocks of the tobacco mosaic virus) or when it exists as multiple "copies" of identical structure. In the latter case, these "copies" may either be arranged in regular aggregates (crystal lattice, helical aggregates), allowing the application of crystallographic reconstruction techniques, or they may be randomly arranged but with an orientational preference, or their arrangement may be entirely random.

$\left({ }^{1}\right)$ That is, to the extent this is permitted by the phase contrast transfer function, which describes the way the projected object potential is imaged under the regime of electron microscopic phase contrast (see, e.g., Hawkes, 1988). 
The rapid progress, in the 70's, in the structural investigation of macromolecules and macromolecular assembles with the electron microscope has benefitted from the existence of a welldeveloped theory of crystal diffraction and 3D reconstruction by Fourier synthesis. However, this theory requires the macromolecules to be rigorously ordered either on a planar lattice (see Stewart, 1988a) or according to helical (see Stewart, 1988b) and spherical symmetries (Crowther, 1971).

When molecules are arranged in a random fashion, on the other hand, two new problems emerge that are not normally tackled by crystallographic data processing techniques: the problem of finding the spatial transformations among the projections appearing in the image ("alignment"), and the problem of reducing structural heterogeneity ("classification").

It is the latter category - of single macromolecules exhibiting no internal symmetries and forming no ordered arrangement - that provided the incentive for the development of entirely new approaches to image analysis and three-dimensional reconstruction (for more extensive treatments, see Frank (1989); Radermacher (1988); Frank et al. $(1985,1988))$. These new approaches will be described in the following. For an authoritative introduction into the well-established method for the reconstruction of planar crystals, the reader is referred to the work by Amos et al. (1982).

\section{Reconstruction from multiple projections of particles with identical structure.}

In a two-dimensional crystal (i.e., a crystal that is a single unit cell thick in the direction normal to the support grid), all molecules occur in a specific, fixed orientation (for the sake of the argument, let us assume that there is only one molecule per unit cell). Therefore, the image of such a crystal is formed by the repeats of the molecule's projection on a regular planar lattice (Fig. 1a). In a Gedankenexperiment, we can create a "generalized crystal" by breaking the crystal up into its constituent molecules and move them into random positions, but maintaining their orientation both relative to the support grid and to one another (Fig. 1b). In the next step, we rotate the molecules in the plane of the grid, so that their projections in the direction perpendicular to the grid remain the same (Fig. 1c). Finally, in the last step of the experiment, we let the molecules assumes arbitrary orientations with respect to the grid (Fig. 1d).

The two scenarios depicted in figures $1 \mathrm{c}$ and $\mathrm{d}$ represent possible experimental situations that lead to radically different data collection geometries and approaches to reconstruction: fixed orientation with random azimuths, leading to the so-called random-conical reconstruction method, and $3 D$-random orientation, leading to a general reconstruction method that faces more substantial obstacles.

3.1 FIXED ORIENTATION, RANDOM AZIMUTHS. - This situation exists when the molecule possesses one distinct face (or several faces) that attaches preferentially to the support film, presenting the advantage that all molecules lying on the same face are equally affected by any anisotropic effects of preparation. When the specimen is prepared by air-drying and negative staining, there are strong forces acting upon the molecule in a direction normal to the plane of the grid, causing the molecule to be flattened. Because of this unavoidable effect, it is not possible to combine projections of differently oriented molecules in the same reconstruction.

Specimens of this kind lend themselves to a very simple method of data collection, and to application of the so-called random-conical method of reconstruction (Radermacher et al, 1987a,b; Radermacher, 1988), whose principle was proposed by Frank et al. (1978; see also Frank and Goldfarb, 1980): briefly, when the specimen grid is tilted by a high angle, then all molecules related to one another by rotation in the plane of the grid form a conical projection series with random azimuths. From this series of projections, the molecule can be reconstructed, provided the com- 

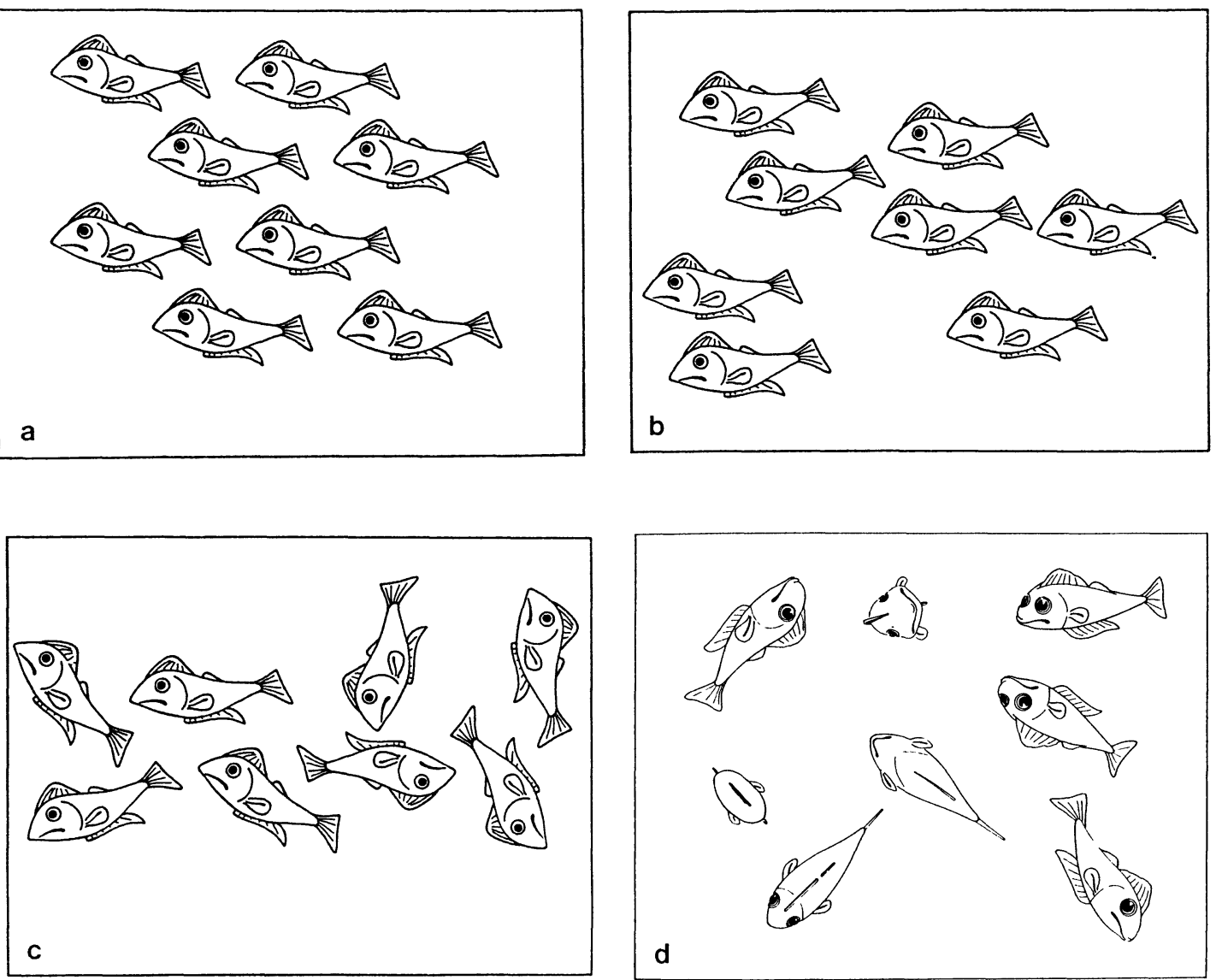

Fig. 1. - The stages in the progression towards disorder in the arrangement of repeats of identical 3D structures: a) two-dimensional crystal: the repeats are identically oriented, and arranged in a regular way; b) translationally disordered crystal: the repeats still have identical orientation but random translations; c) translationally and rotationally disordered arrangement: only the orientation with respect to the "support grid" is preserved; i.e., the structure still presents the same projection; d) totally random arrangement in which even the viewing direction changes, so that different projections co-exist. (a-c from Frank and Goldfarb (1980), reproduced with pemission by Springer-Verlag)

plete azimuthal range is filled without major gaps. The azimuthal angles of the projections are obtained by the analysis of a companion micrograph that shows the specimen grid in the untilted position, using translational and rotational alignment techniques (Frank et al, 1978, 1981).

Typically, several hundred projections go into a single reconstruction. The large number is required for an even statistical coverage of the 360-degree azimuthal range, and to achieve a high signal-to-noise ratio in the $3 \mathrm{D}$ result. Figure 2 shows an example for a reconstruction using this technique: the calcium release channel of the sarcoplasmic reticulum (Wagenknecht et al., 1989).

Upon close inspection, it can be seen that the molecules within the specimen field vary in appearence: they may occur in different orientations, or they may be damaged, deformed, or unevenly stained. Some type of classification is clearly needed to divide the molecule population into homogeneous groups with uniform appearence and a "mixed bag" of rejects, i.e., molecules exhibiting artifacts. Only within a homogeneous group, the reconstruction scheme described above 


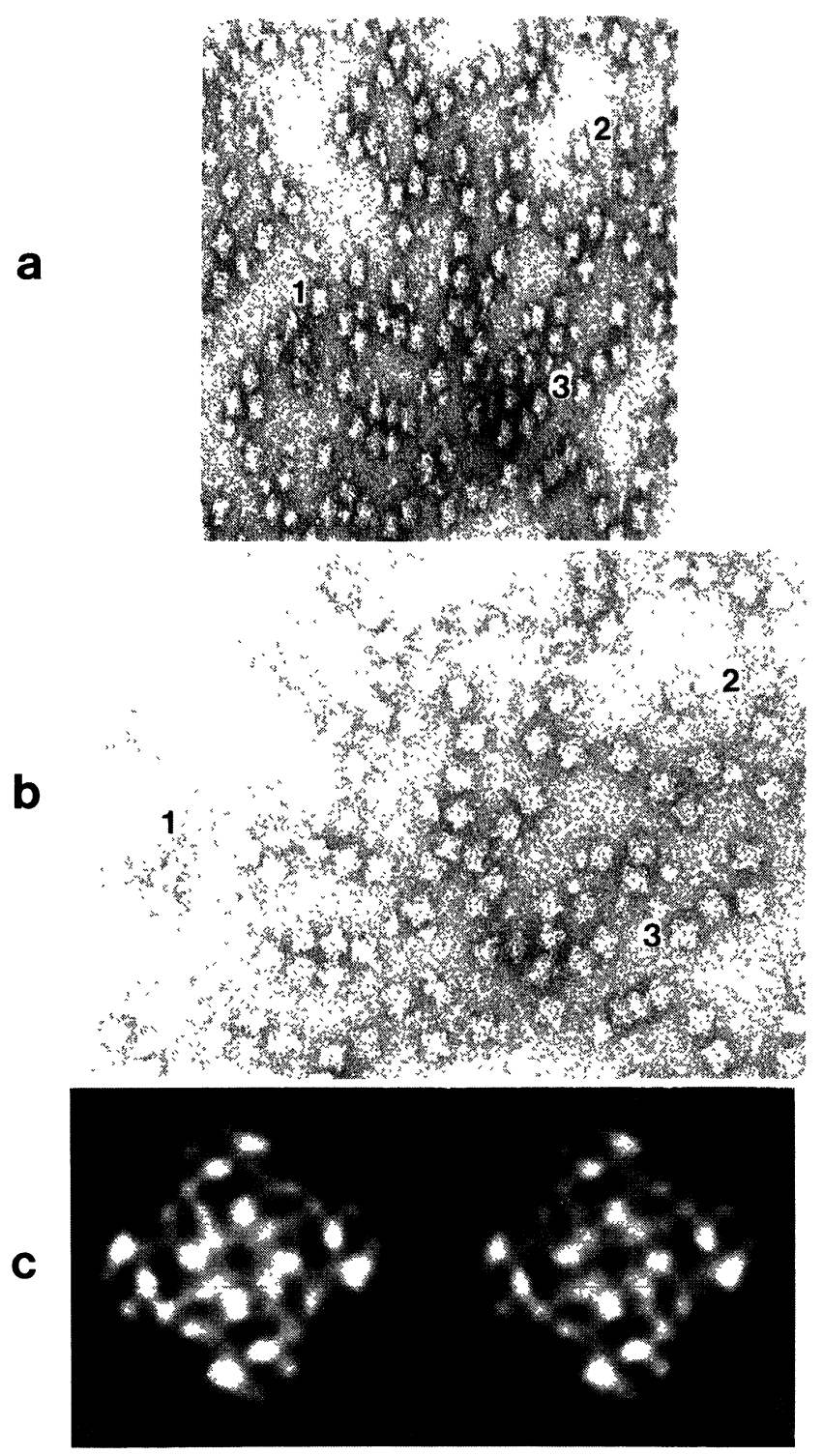

Fig. 2. - Three-dimensional reconstruction of the calcium release channel of the sarcoplasmic reticulum, utilizing common orientation and random azimuths of the particle for the random-conical method of data collection. The negative staining method (uranyl acetate) was used, with the specimen sandwiched between two thin carbon layers. Low-dose (approx. $10 \mathrm{el} / \mathrm{A}$ ) technique was used for recording. a) Specimen grid tilted by $50^{\circ} ;$ b) the same grid without tilt. (Numbers are inserted to mark corresponding particles for easy comparison). The micrograph in (a) contains the equivalent of a conical projection set with a cone angle of $50^{\circ}$. c) Averages over comparable numbers of $0^{\circ}$-particle projections ( 140 in each case) demonstrating the high degree of reproducibility ( $25 \AA$ according to phase residual analysis). d)-f) Stereo-pairs of computed solid model representation of the channel complex reconstructed from 280 projections of the type shown in (a). Additionally, fourfold symmetry was imposed. BP, back plate; PL, peripheral lobe. g) The channel complex cut open to reveal radial channels RC going from central cavity CC to peripheral vestibules PV. [from Wagenknecht et al. (1989); reproduced with permission by MacMillan Magazines, Ltd.] Scale bar = $100 \AA$. 


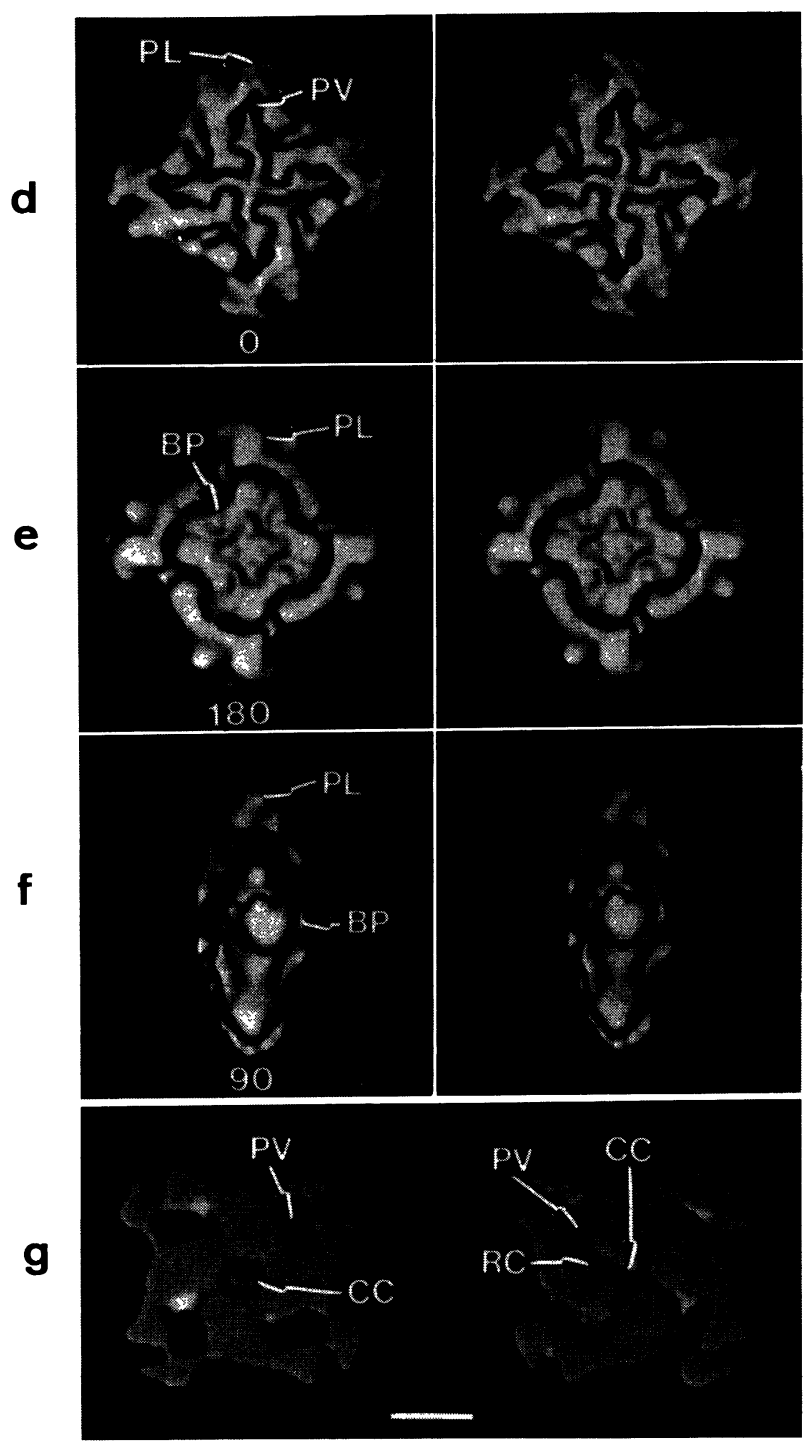

Fig. 2. - (continued)

will lead to a meaningful answer. Classification is intrinsically hierarchic, working from the coarse features to the fine. We have found it practical to do the coarsest step of classification (e.g., side views versus frontal views; complete versus fragmented molecules) by visual inspection, while making any finer divisions (e.g., selection of molecules with the same orientation from those in a range of orientations, or division of molecules with similar outline into subgroups according to interior features) requires a quantitative, at least partially automated approach. Techniques for this latter step, based on a multivariate statistical analysis of the data set, are now in routine use (Van Heel and Frank, 1981; Van Heel, 1984; 1989). A review of automatic classification techniques is found in (Frank, 1990). 
Recently, we found that the random-conical method of reconstruction can still be used for iceembedded particles (Frank et al., 1991; Radermacher et al. 1992; Boisset et al, 1992) even though the decreased signal-to-noise ratio inherent to cryo-images poses a bigger hurdle to correlation and classification techniques. Special algorithms for alignment and reconstruction have been developed for this purpose (Penczek et al., 1992). Thus it is now possible to obtain information on the interior mass distribution of macromolecular complexes (see putative localization of ribosomal RNA within the Escherichia coli ribosome by Frank et al., 1991).

3.2 THREE-DIMENSIONAL RANDOM ORIENTATIONS. - In principle, macromolecules may possess entirely random orientations, thus precluding use of the random-conical reconstruction method. Provided that its 3D shape is equally preserved for all orientations, such a molecule can be reconstructed from its projections. The obvious challenge in this situation is to find the relative angles of all projections.

What is the prospect of encountering these conditions (random orientation, no shape change)? Negative staining is known to deform the particle nonuniformly, and thus precludes the use of random orientations in a single reconstruction (see Carazo et al., 1989). Embedding in ice, on the other hand, which most likely avoids the deformation of shape, appears to produce strong orientational preferences: e.g., in our experience with the Escherichia coli ribosome (Frank $e t$ al., 1991) and its large subunit (Wagenknecht et al., 1988, Radermacher et al., 1992a), calcium release channel (Radermacher et al., 1922b), and alpha-2 macroglobulin (Boisset et al., 1992). This experience seems to suggest that the number of structures amenable to reconstruction from a random projection set is probably limited.

Back to the challenge of finding the directions of projection: Provencher and Vogel's method of reconstruction (1988), which incorporates an angle search, stops short of a solution to this problem because it requires approximate starting angles. In fact, if we consider the goal: that we wish to relate to one another the projections of an unknown structure into unknown directions, we may be facing one of the most complex of pattern recognition and artificial intelligence yet formulated. Although a number of theoretical approaches to this problem have been proposed (Goncharev et al. 1988; Van Heel, 1987), based in principle on the method of common lines (Crowther et al., 1970), there is currently no indication as to which method will work with the extremely noisy images obtained with frozen-hydrated specimens. The development of methods for relative angle search and various approaches to the classification of extremely noisy data is therefore one of the important issues in advancing 3D molecular imaging with the electron microscope.

\section{The problem of the missing angular range.}

Depending upon the data collection method used, we may be faced with a problem unique to electron microscopy: the angular range accessible by tilting is normally limited because of the space limitations in the objective lens and because of the "slab" geometry of normal specimens, which leads to excessive pathlengths at high angles. As a consequence of this missing data range, the reconstruction has artifacts that interfere with its interpretation: each point of the structure is smeared out in the direction of the missing data (anisotropic resolution), and the structure as a whole is elongated in this direction. (This problem can of course be avoided when the requirements of fully random data collection are fulfilled; see above discussion.)

In the random conical reconstruction method, the missing data range has the shape of a cone whose opening angle is $2 *$ (90 - tilt angle). If more than one particle orientation is available, then several reconstructions can be combined to cover the missing cone. This approach has been successfully used in the case of the ribosome (Frank et al, 1991; Penczek et al., 1992). 
An entirely different solution to the problem of the missing data is provided by restoration methods, which make use of information not taken into account in the normal reconstruction procedure, such as value and shape boundaries. Two of these methods, the Maximum Entropy Restoration (Lawrence et al., 1989; Barth et al., 1989) and the method of Projection onto Convex Sets (POCS (Carazo and Carrascosa, 1987a,b; Zhang et al., 1990) are currently being actively pursued in electron microscopy. The latter method is based on a set theoretical statement of the problem in Hilbert space (or its finite equivalent for discrete data): a constraint can be represented by the set of all functions obeying it. If several constraints are known to hold, then the solution should be contained in the intersection of the corresponding sets. POCS rests on a property of closed and convex sets: for such sets it is possible to find a solution that is both close to the conventional reconstruction (obtained by weighted back-projection) and lies in the intersection, i.e., fulfills all constraints.

The capability of this method to retrieve the undistorted object is demonstrated by a model computation (Fig. 3; Zhang, 1992): here a limited tilt series was computed, and from this a conventional reconstruction was obtained. After application of the POCS scheme, the object was retrieved, with the quality of the result obviously dependent on the noise level.

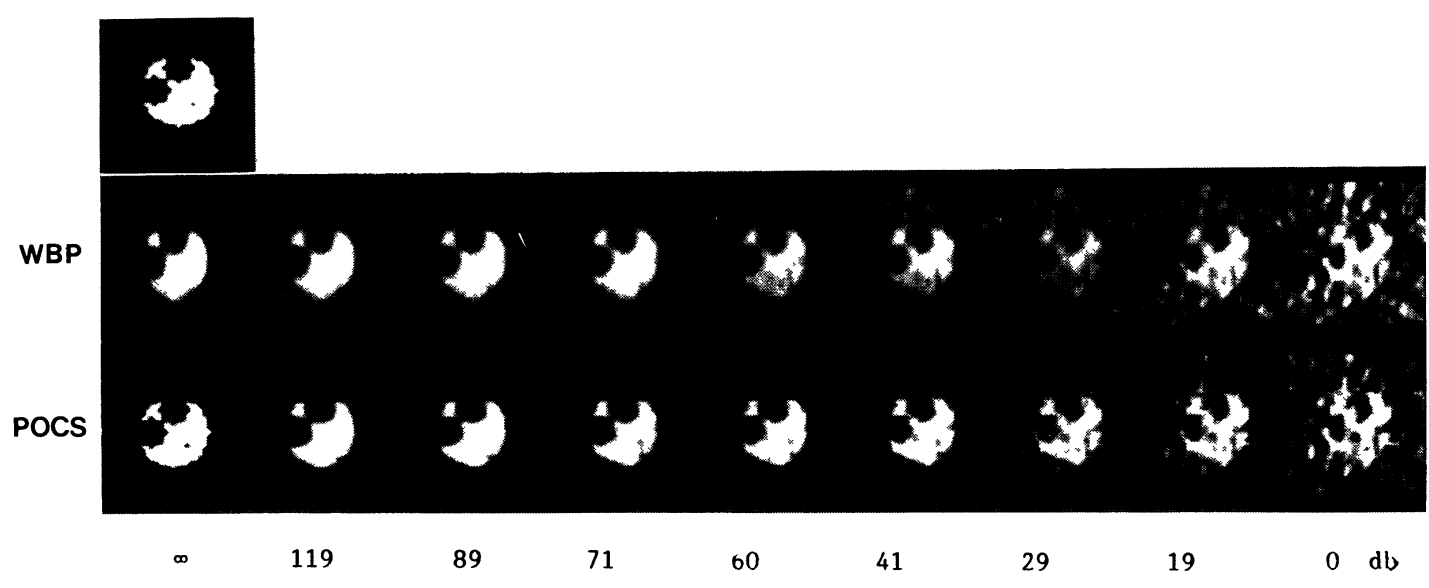

Fig. 3. - Model computation to test the POCS restoration scheme. A phantom (top of figure) was projected into 11 angles in the range $+/-60$ degrees, and noise was added. From this noisy projection set, a reconstruction was obtained by weighted back-projection (WBP). Subsequently, POCS was applied to the WBP reconstruction. On the panel, the noise increases in horizontal direction, from infinite $\mathrm{db}$ to $0 \mathrm{db}$. The following constraints were used in 50 POCS iteractions: Non-negativity, Upper density bound, Energy bound, Finite support (as defined by a binary mask), and Replace (in the measured region of the Fourier transform). The results show the ability of POCS to retrieve the object perfectly in the noise-free situation (infinite $\mathrm{S} / \mathrm{N}$ ratio), and to limit the artifacts due to the missing angular range when noise is present $(S / N$ ratio between 0 and 119). (From Zhang, 1992).

\section{Outlook.}

The new possibility of obtaining 3D images of macromolecules even when they occur in singleparticle form has opened up many new avenues of research. When this prospect first appeared on 
the horizon, it was greeted with the words "the sky would be the limit". After our first experience with structural variability, specimen flattening, and the struggle to fill the missing cone in Fourier space, we would probably put the limit closer to earth. Nevertheless, the few years during which the single particle reconstruction technique has been in existence have yielded a wealth of new information on the architecture of macromolecular assemblies difficult to obtain in crystal form.

\section{Acknowledgements.}

This work was supported, in part, by grants NIH 1R01 GM29169 and 1 S10 RR 03998.

\section{References}

AMOS L.A., HENDERSON R. and UNWIN P.N.T., Three-dimensional structure determination by electron microscopy of two-dimensional crystals Prog. Biophys. Mol. Biol. 39 (1982) 183-231.

BARTH M., BRYAN R.K. and HEGERL R., Approximation of missing-cone data in 3D electron microscopy, Ultramicroscopy 31 (1989) 365-378.

Boisset N., Grassucci R., MOtTa G., LAMY L., Radermacher M., TAVEAU J.C., LiU W., Frank J. and LAMY J.N., Three-dimensional immunoelectron microscopy of Androctonus australis hemocyanin: the location of monoclonal Fab fragments specific for subunit Aa6, Proc. XII Intl. Congr. Electr. Microsc. (1992) in press.

CARAZO J.M. and CARRASCOSA J.L., Information recovery in missing angular data cases: an approach by the convex projections method in three dimensions, J. Microsc. 145 (1987a) 23-43.

CARAZO J.M. and CARRASCOSA J.L., Restoration of direct Fourier three-dimensional reconstructions of crystalline specimens by the method of convex projections J. Microsc. 145 (1987b) 159-177.

CARAZO J.M., WAGENKNECHT T. and FRANK J., Variations of the three-dimensional structure of the Escherichia coli ribosome in the range of overlap views, Biophys. J. 55 (1989) 465-477.

CROWTHER R.A., DEROSIER D.J. and KLUG A., The reconstruction of a three-dimensional structure from projections and its application to electron microscopy, R. Soc. London Proc. A317 (1970) 319-340.

CROWTHER R.A., Procedures for three-dimensional reconstruction of spherical viruses by Fourier synthesis from electron micrographs, Philos. Trans. R. Soc. (Lond.) B 261 (1971) 221-230.

DEROSIER D.J. and KLUG A., Reconstruction of three-dimensional structures from electron micrographs, Nature 217 (1968) 130-134.

Dubochet J., AdRian M., Chang J.-J., Homo J.-C., Lepault J., McDowall A.W. and Schultz P., Cryoelectron microscopy of vitrified specimen Quart. Rev. Biophys. 21 (1988) 129-228.

FRANK J. and GOLDFARB W., Methods for averaging of single molecules and lattice fragments. In: Electron Microscopy at Molecular Dimensions, W. Baumeister and W. Vogell Eds. (Springer-Verlag, Berlin, 1980) pp.261-269.

FRANK J., Three-dimensional imaging techniques in electron microscopy, BioTechnology 7 (1989) 164-173.

FRANK J., GOLDFARB W., EISENBERG D. and BAKER T.S., Reconstruction of glutamine synthetase using computer averaging, Ultramicroscopy 3 (1978) 283-290.

FRANK J., VERSCHOOR A. and BOUBLIK M., Computer averaging of electron micrographs of 40S ribosomal subunits, Science 214 (1981) 1353-1355.

FRANK J., VERSCHOOR A. and WAGENKNECHT T., Computer processing of electron microscopic images of single macromolecules. In : New Methodologies in Studies of Protein Configuration, T.T. Wu Ed. (Van Nostrand Reinhold, New York, 1985) pp. 36-89.

FRANK J., RADERMACHER M., WAGENKNECHT T. and VERSCHOOR A., Studying ribosome structure by electron microscopy and computer-image processing Methods in Enzymology 164 (1988) 3-35.

GILBERT P.F.C., Iterative methods for the three-dimensional reconstruction of an object from projections, $J$. Theor. Biol. 36 (1972) 105-117.

GONCHAREV A.B., VAINSHTEIN B.K., RYSKIN A.I. and VAGIN A.A., Three-dimensional reconstruction of arbitrarily oriented identical particles from their electron photomicrographs, Sov. Phys. Crystallogr. 32 (1988) 504-509. 
HAWKES P.W., Image processing based on the linear theory of image formation. In: Computer Processing of Electron Microscope Images, P.W. Hawkes Ed. (Springer-Verlag Berlin, 1980) pp. 1-33.

HOPPE W., GaSSMANN J., HunSMANN N., SCHRAMM J. and STURM M., Three-dimensional reconstruction of individual negatively stained yeast fatty-acid synthetase molecules from tilt series in the electron microscope, Hoppe-Seyler's Z. Physiol. Chem. 355 (1974) 1483-1487.

LAWRENCE M.C., JAFFER M.A. and SEWELL B.T., The application of the maximum entropy method to electron microscopic tomography, Ultramicroscopy 31 (1989) 285-302.

PENCZEK P., RADERMACHER M. and FRANK J., Three-dimensional reconstruction of single particles embedded in ice. Ultramicroscopy 40 (1992) 33-53.

PROVENCHER S.W. and VOGEL R.H., Three-dimensional reconstruction from electron micrographs of disordered specimens. I. Method, Ultramicroscopy 25 (1988) 209-222.

RADERMACHER M., Three-dimensional reconstruction of single particles from random and nonrandom tilt series, J. Electron Microsc. Techn. 9 (1988) 359-394.

RADERMACHER M., WAGENKNECHT T., VERSCHOOR A. and FRANK J., Three-dimensional reconstruction from a single-exposure random conical tilt series applied to the 50S ribosomal subunit of Escherichia coli, J. Microsc. 146 (1987a) 113-136.

RADERMACHER M., WAGENKNECht T., VERSCHOOR A. and Frank J., Three-dimensional structure of the large subunit from Escherichia coli, EMBO J. 6 (1987) 1107-1114.

RADERMACHER M., SRIVASTAVA S. and FRANK J., The structure of the 50 S ribosomal subunit from $E$. coli in frozen hydrated preparation reconstructed with SECRET, Proc. XII Intl. Congr. Electr. Microsc. (1992a) in press.

Radermacher M., Wagenknecht T., Grassucci R., Frank J., Chadwick C. and Fleischer S., CryoEM of the native structure of the calcium release channel/ryanodine receptor from sarcoplasmic reticulum, Biophys. J. 61 (1982b) 1-6.

STEWART M., Introduction to the computer image processing of electron micrographs of two-dimensionally ordered biological structures, J. Electron Microsc. Techn. 9 (1987a) 301-324.

STEWART M., Computer image processing of electron micrographs of biological structures with helical symmetry J. Electron Microsc. Techn. 9 (1988b) 325-358.

VAN HEEL M. and FRANK J., Use of multivariate statistics in analyzing the images of biological macromolecules. Ultramicroscopy 6 (1981) 187-194.

VAN HEEL M., Angular reconstitution: a-posteriori assignment of projection directions for 3D reconstruction, Ultramicroscopy 21 (1987) 111-124.

WAGENKNECHT T., GRASSUCCI R. and FRANK J., Electron microscopy and computer image processing of ice-embedded large ribosomal subunits from Escherichia coli, J. Mol. Biol. 199 (1988) 137-147.

WAGENKNECHT T., GRASSUCCI R., FRANK J., SAITO A., INUI M. and FLEISCHER S. (1989) Three-dimensional architecture of the calcium channel/foot structure of sarcoplasmic reticulum, Nature 338(1989) 167170.

ZHANG N.Y., A new method of 3D reconstruction and restoration in electron microscopy: Least squares method combined with projection onto convex sets (LSPOCS), Thesis, State University of New York at Albany (1992).

ZHANG N.Y., MCEWEN B.F. and FRANK J., On the use of POCS for restoring the "missing wedge" in electron tomography. Proc. XII Int. Congr. Electr. Microscopy, Vol 1 (1990) pp.520-521.

ZWICK M. and ZEITLER E., Image reconstruction from projections, Optik 38 (1973) 550-565. 